\title{
MODIFIED PROXIMAL POINT ALGORITHM FOR MINIMIZATION AND FIXED POINT PROBLEM IN CAT(0) SPACES
}

\author{
Godwin Chidi Ugwunnadi \\ Department of Mathematics, University of Eswatini, \\ Private Bag 4, Kwaluseni, Eswatini \\ Department of Mathematics and Applied Mathematics, \\ Sefako Makgato Health Science University, \\ P.O. Box 94, Pretoria 0204, South Africa \\ ugwunnadi4u@yahoo.com
}

\begin{abstract}
In this paper, we study modified-type proximal point algorithm for approximating a common solution of a lower semi-continuous mapping and fixed point of total asymptotically nonexpansive mapping in complete $\mathrm{CAT}(0)$ spaces. Under suitable conditions, some strong convergence theorems of the proposed algorithms to such a common solution are proved.
\end{abstract}

Keywords: Proximal point algorithm, Total asymptotically nonexpansive mapping, Fixed point, $\triangle$ convergence, Strong convergence, CAT $(0)$ space.

\section{Introduction}

In recent years, much attention has been given to develop several iterative methods including the proximal point algorithms (PPA) which was suggested by Martinet [26] for solving convex optimization problems which was extensively developed by Rockafellar [28] in the context of monotone variational inequalities. The main idea of this method consists of replacing the initial problem with a sequence of regularized problems, so that each particular auxiliary problem can be solved by one of the well-known algorithms. Quiet number of different method of proximal point algorithm have been proposed and studied from the classical linear spaces such as Euclidean spaces, Hilbert spaces, and Banach spaces to the setting of manifolds (see [5, 6, 13, 18, 20, 26, 28]).

Recently, the classical proximal point algorithms have been extended from linear spaces such as Hilbert spaces or Banach spaces to the setting of nonlinear version.

In 2013, Bačák [6] introduced the PPA in a $\operatorname{CAT}(0)$ space $(X, d)$ as follows: $x_{1} \in X$ and

$$
x_{n+1}=\underset{y \in X}{\arg \min }\left(f(y)+\frac{1}{2 \lambda_{n}} d^{2}\left(y, x_{n}\right)\right), \quad \forall n \geq 1,
$$

where $\lambda_{n}>0, \forall n \geq 1$. It was shown that if $f$ has a minimizer and

$$
\sum_{n=1}^{\infty} \lambda_{n}=\infty
$$

then the sequence $\left\{x_{n}\right\} \triangle$-converges to its minimizer [5].

It is a known fact that iterative methods for finding fixed points of nonexpansive mappings have received vast investigations due to its extensive applications in a variety of applied areas of inverse 
problem, partial differential equations, image recovery, and signal processing; see $[2,5,8,15,21]$ and the references therein.

Fixed-point theory in CAT(0) spaces was first studied by Kirk [22, 23]. He showed that every nonexpansive (single-valued) mapping defined on a bounded closed convex subset of a complete CAT(0) space always has a fixed point. Since then, the fixed-point theory for single-valued and multivalued mappings in CAT(0) spaces has been rapidly developed.

Recently, Suparatulatorn et al. [29] presented a new modified proximal point algorithm for solving the minimization of a convex function and the fixed points of nonexpansive mappings in CAT(0) spaces. Chang et al. [12] proved some strong convergence theorems of the PPA to a common fixed point of asymptotically nonexpansive mappings and to minimizers of a convex function in $\operatorname{CAT}(0)$ spaces.

Let $C$ be a nonempty subset of a complete CAT(0) space $X$ and $T$ a mapping from $C$ into itself. Then, a point $x \in C$ is called a fixed point of $T$ if $T x=x$. We denote by $F(T)$ the set of all the fixed points of $T$. A mapping $T$ from $C$ into itself is said to be:

(N) nonexpansive if $d(T x, T y) \leq d(x, y)$ for all $x, y \in C$;

(AN) asymptotically nonexpansive, if there is a sequence $\left\{u_{n}\right\} \subseteq[0, \infty)$ with $u_{n} \rightarrow 0$ as $n \rightarrow \infty$ such that

$$
d\left(T^{n} x, T^{n} y\right) \leq\left(1+u_{n}\right) d(x, y), \quad \forall n \geq 1, \quad x, y \in C ;
$$

(UL) uniformly $L$-Lipschitzian, if there exists a constant $L>0$ such that

$$
d\left(T^{n} x, T^{n} y\right) \leq L d(x, y), \quad \forall n \geq 1, \quad x, y \in C .
$$

The concept of total asymptotically nonexpansive mappings was first introduced by Alber et al. [1]. A mapping $T: C \rightarrow C$ is said to be total asymptotically nonexpansive mapping if there exists nonnegative sequences $\left\{\mu_{n}\right\},\left\{\nu_{n}\right\}$ with $\mu_{n} \rightarrow 0, \nu_{n} \rightarrow 0$ as $n \rightarrow \infty$ and a strictly increasing continuous function $\zeta:[0, \infty) \rightarrow[0, \infty)$ with $\zeta(0)=0$ such that

$$
d\left(T^{n} x, T^{n} y\right) \leq d(x, y)+\nu_{n} \zeta(d(x, y))+\mu_{n}, \quad \forall n \geq 1, \quad x, y \in C .
$$

Remark 1. From the definitions, it is known that each nonexpansive mapping is asymptotically nonexpansive mapping with sequence $\left\{u_{n}=0\right\}$, and each asymptotically nonexpansive mapping is $\left(\left\{\mu_{n}\right\},\left\{\nu_{n}\right\}, \zeta\right)$-total asymptotically nonexpansive mapping with $\mu_{n}=0, \nu_{n}=u_{n}, \forall n \geq 1$ and $\zeta(t)=t, t \geq 0$. But the opposite may not be true for each of them in a general sense. Furthermore, every asymptotically nonexpansive mapping is a uniformly $L$-Lipschitzian mapping with

$$
L=\sup _{n \geq 1}\left(1+u_{n}\right)
$$

Motivated and inspired by the above works, in this paper, we study a modified algorithm for proximal point and fixed point of total asymptotically nonexpansive mapping in $\operatorname{CAT}(0)$ space. Strong convergence of this algorithm is proved. Our method of proof is different from the method in Chang et al. [12].

\section{Preliminaries}

Let $(X, d)$ be a metric space and $x, y \in X$ with $d(x, y)=l$. A geodesic path from $x$ to $y$ is an isometry $c:[0, l] \rightarrow X$ such that $c(0)=x$ and $c(l)=y$. The image of a geodesic path is 
called a geodesic segment. A metric space $X$ is a (uniquely) geodesic space, if every two points of $X$ are joined by only one geodesic segment. A geodesic triangle $\triangle\left(x_{1}, x_{2}, x_{3}\right)$ in a geodesic space $X$ consists of three points $x_{1}, x_{2}, x_{3}$ of $X$ and three geodesic segments joining each pair of vertices. A comparison triangle of a geodesic triangle $\triangle\left(x_{1}, x_{2}, x_{3}\right)$ is the triangle $\bar{\triangle}\left(x_{1}, x_{2}, x_{3}\right):=$ $\triangle\left(\bar{x}_{1}, \bar{x}_{2}, \bar{x}_{3}\right)$ in the Euclidean space $\mathbb{R}^{2}$ such that

$$
d\left(x_{i}, x_{j}\right)=d_{\mathbb{R}^{2}}\left(\bar{x}_{i}, \bar{y}_{j}\right), \quad \forall i, j=1,2,3 .
$$

A geodesic space $X$ is a $\mathrm{CAT}(0)$ space, if for each geodesic triangle $\triangle\left(x_{1}, x_{2}, x_{3}\right)$ in $X$ and its comparison triangle $\bar{\triangle}:=\triangle\left(\bar{x}_{1}, \bar{x}_{2}, \bar{x}_{3}\right)$ in $\mathbb{R}^{2}$, the $\operatorname{CAT}(0)$ inequality $d(x, y) \leq d_{\mathbb{R}^{2}}(\bar{x}, \bar{y})$ is satisfied for all $x, y \in \triangle$ and $\bar{x}, \bar{y} \in \bar{\triangle}$.

A thorough discussion of these spaces and their important role in various branches of mathematics are given in $[9,10]$. Let $x, y \in X$ and $\lambda \in[0,1]$, we write $\lambda x \oplus(1-\lambda) y$ for the unique point $z$ in the geodesic segment joining from $x$ to $y$ such that

$$
d(z, x)=(1-\lambda) d(x, y) \quad \text { and } \quad d(z, y)=\lambda d(x, y) .
$$

We also denote by $[x, y]$ the geodesic segment joining from $x$ to $y$, that is,

$$
[x, y]=\{\lambda x \oplus(1-\lambda) y: \lambda \in[0,1]\} .
$$

A subset $C$ of a $\operatorname{CAT}(0)$ space is convex if $[x, y] \subseteq C$ for all $x, y \in C$.

Berg and Nikolaev [7] introduced the concept of an inner product-like notion (quasilinearization) in complete $\mathrm{CAT}(0)$ spaces to resolve these difficulties as follows:

Let denote a pair $(a, b) \in X \times X$ by $\overrightarrow{a b}$ and call it a vector. The quasilinearization is a map $\langle.,\rangle:.(X \times X) \times(X \times X) \rightarrow \mathbb{R}$ defined by

$$
\langle\overrightarrow{a b}, \overrightarrow{c d}\rangle=\frac{1}{2}\left(d^{2}(a, d)+d^{2}(b, c)-d^{2}(a, c)-d^{2}(b, d)\right), \quad \forall a, b, c, d \in X .
$$

It is easily seen that $\langle\overrightarrow{a b}, \overrightarrow{c d}\rangle=\langle\overrightarrow{c d}, \overrightarrow{a b}\rangle,\langle\overrightarrow{a b}, \overrightarrow{c d}\rangle=-\langle\overrightarrow{b a}, \overrightarrow{c d}\rangle$ and $\langle\overrightarrow{a x}, \overrightarrow{c d}\rangle+\langle\overrightarrow{x b}, \overrightarrow{c d}\rangle=\langle\overrightarrow{a b}, \overrightarrow{c d}\rangle$ for all $a, b, c, d \in X$. We say that $X$ satisfies the Cauchy-Schwarz inequality if

$$
\langle\overrightarrow{a b}, \overrightarrow{c d}\rangle \leq d(a, b) d(c, d)
$$

for all $a, b, c, d \in X$. It is known that a geodesically connected metric space is a CAT(0) space if and only if it satisfies the Cauchy-Schwarz inequality (see [7]).

Lemma 1 [16]. Let $X$ be a $\mathrm{CAT}(0)$ space, $x, y, z \in X$ and $\lambda \in[0,1]$. Then

$$
d(\lambda x \oplus(1-\lambda) y, z) \leq \lambda d(x, z)+(1-\lambda) d(y, z) .
$$

Lemma 2 [16]. Let $X$ be a $\mathrm{CAT}(0)$ space, $x, y, z \in X$ and $\lambda \in[0,1]$. Then

$$
d^{2}(\lambda x \oplus(1-\lambda) y, z) \leq \lambda d^{2}(x, z)+(1-\lambda) d^{2}(y, z)-\lambda(1-\lambda) d^{2}(x, y) .
$$

Lemma 3 [14]. Let $X$ be a $\mathrm{CAT}(0)$ space, $x, y, z \in X$ and $\lambda \in[0,1]$. Then

$$
d^{2}(\lambda x \oplus(1-\lambda) y, z) \leq \lambda^{2} d^{2}(x, z)+(1-\lambda)^{2} d^{2}(y, z)+2 \lambda(1-\lambda)\langle\overrightarrow{x z}, \overrightarrow{y z}\rangle .
$$


Let $\left\{x_{n}\right\}$ be a bounded sequence in a complete CAT(0) space $X$. For $x \in X$, we set

$$
r\left(x,\left\{x_{n}\right\}\right)=\limsup _{n \rightarrow \infty} d\left(x, x_{n}\right) .
$$

The asymptotic radius $r\left(\left\{x_{n}\right\}\right)$ of $\left\{x_{n}\right\}$ is given by

$$
r\left(\left\{x_{n}\right\}\right)=\inf \left\{r\left(x,\left\{x_{n}\right\}\right): x \in X\right\},
$$

and the asymptotic center $A\left(\left\{x_{n}\right\}\right)$ of $\left\{x_{n}\right\}$ is the set

$$
A\left(\left\{x_{n}\right\}\right)=\left\{x \in X: r\left(x,\left\{x_{n}\right\}\right)=r\left(\left\{x_{n}\right\}\right)\right\} .
$$

It is well known that in a $\operatorname{CAT}(0)$ space $A\left(\left\{x_{n}\right\}\right)$ consists of exactly one point (see [15, Proposition 7]).

Lemma 4 [24]. Every bounded sequence in a complete CAT(0) space always has a $\triangle$-convergent subsequence.

Lemma 5 [19]. Let $X$ be a complete CAT(0) space, $\left\{x_{n}\right\}$ be a sequence in $X$ and $x \in X$. Then $\left\{x_{n}\right\} \triangle$-converges to $x$ if and only if $\limsup _{n \rightarrow \infty}\left\langle\overrightarrow{x x_{n}}, \overrightarrow{x y}\right\rangle \leq 0$ for all $y \in C$.

A function $f: C \rightarrow(-\infty, \infty]$ defined on a convex subset $C$ of a $\operatorname{CAT}(0)$ space is convex if, for any geodesic

$$
[x, y]:=\left\{\gamma_{x, y}(\lambda): 0 \leq \lambda \leq 1\right\}:=\{\lambda x \oplus(1-\lambda) y: 0 \leq \lambda \leq 1\}
$$

joining $x, y \in C$, the function $f \circ \gamma$ is convex, i.e.

$$
f\left(\gamma_{x, y}(\lambda)\right):=f(\lambda x \oplus(1-\lambda) y) \leq \lambda f(x)+(1-\lambda) f(y) .
$$

For examples of convex functions in $\operatorname{CAT}(0)$, see [12]. For any $\lambda>0$, define the Moreau-Yosida resolvent of $f$ in $\operatorname{CAT}(0)$ space $X$ as

$$
J_{\lambda}(x)=\underset{y \in X}{\arg \min }\left[f(y)+\frac{1}{2 \lambda} d^{2}(y, x)\right], \quad \forall x \in X .
$$

Let $f: X \rightarrow(-\infty, \infty]$ be a proper convex and lower semi-continuous function. It is shown in [3] that the set $F\left(J_{\lambda}\right)$ of fixed points of the resolvent associated with $f$ coincides with the set $\operatorname{argmin}_{y \in X} f(y)$ of minimizers of $f$. Also for any $\lambda>0$, the resolvent $J_{\lambda}$ of $f$ is nonoexpansive [17].

Lemma 6 (Sub-differential inequality [4]). Let $(X, d)$ be a complete CAT $(0)$ space and $f: X \rightarrow(-\infty, \infty]$ be proper convex and lower semi-continuous. Then, for all $x, y \in X$ and $\lambda>0$, the following inequality holds:

$$
\frac{1}{2 \lambda} d^{2}\left(J_{\lambda} x, y\right)-\frac{1}{2 \lambda} d^{2}(x, y)+\frac{1}{2 \lambda} d^{2}\left(x, J_{\lambda} x\right)+f\left(J_{\lambda} x\right) \leq f(y) .
$$

Lemma $7[17,27]$ (The resolvent identity). Let $(X, d)$ be a complete CAT $(0)$ space and $f: X \rightarrow(-\infty, \infty]$ be proper convex and lower semi-continuous. Then the following identity holds:

$$
J_{\lambda} x=\left(\frac{\lambda-\mu}{\lambda} J_{\lambda} x \oplus \frac{\mu}{\lambda} x\right)
$$

for all $x \in X$ and $\lambda>\mu>0$. 
Lemma 8 [11]. If $C$ is a closed convex subset of a complete CAT(0) space $X$ and $T: C \rightarrow X$ be a uniformly L-Lipschitzian and total asymptotically nonexpansive mappings. Let $\left\{x_{n}\right\}$ be a bounded sequence in $C$ such that $x_{n} \rightarrow p$ and

$$
\lim _{n \rightarrow \infty} d\left(x_{n}, T x_{n}\right)=0 .
$$

Then $T p=p$.

Lemma 9 [25]. Let $\left\{a_{n}\right\}$ be a sequence of real numbers such that there exists a subsequence $\left\{n_{i}\right\}$ of $\{n\}$ such that $a_{n_{i}}<a_{n_{i}+1}$ for all $i \in \mathbb{N}$. Then there exists a nondecreasing sequence $\left\{m_{k}\right\} \subset \mathbb{N}$ such that $m_{k} \rightarrow \infty$ and the following properties are satisfied by all (sufficiently large) numbers $k \in \mathbb{N}$

$$
a_{m_{k}} \leq a_{m_{k}+1} \quad \text { and } \quad a_{k} \leq a_{m_{k}+1}
$$

In fact,

$$
m_{k}=\max \left\{j \leq k: a_{j}<a_{j+1}\right\} .
$$

Lemma 10. (Xu, [30]) Let $\left\{a_{n}\right\}$ be a sequence of nonnegative real numbers satisfying the following relation:

$$
a_{n+1} \leq\left(1-\alpha_{n}\right) a_{n}+\alpha_{n} \sigma_{n}+\gamma_{n}, \quad n \geq 0
$$

where, $(i)\left\{\alpha_{n}\right\} \subset[0,1], \sum \alpha_{n}=\infty ; \quad$ (ii) limsup $\sigma_{n} \leq 0 ; \quad$ (iii) $\gamma_{n} \geq 0 ; \quad(n \geq 0), \sum \gamma_{n}<\infty$. Then, $a_{n} \rightarrow 0$ as $n \rightarrow \infty$.

\section{Main Result}

Theorem 1. Let $X$ be a complete $\mathrm{CAT}(0)$ space and $C$ be a nonempty closed convex subset of $X$. Let $f: C \rightarrow(-\infty, \infty]$ be a proper convex and lower semi-continuous function and $T: C \rightarrow C$ be L-Lipschitzian and total asymptotically nonexpansive mappings with $\left\{u_{n}\right\},\left\{v_{n}\right\}$ and mappings $\zeta:[0, \infty) \rightarrow[0, \infty)$ satisfying $\sum_{n=1}^{\infty} u_{n}<\infty$ and $\sum_{n=1}^{\infty} v_{n}<\infty$ such that

$$
\Omega:=F(T) \bigcap \underset{y \in C}{\arg \min } f(y) \neq \emptyset .
$$

Let $\left\{x_{n}\right\}_{n=1}^{\infty}$ be a sequence generated by $x_{1}=w \in C$ chosen arbitrarily,

$$
\left\{\begin{array}{l}
z_{n}=\underset{y \in C}{\arg \min }\left[f(y)+\frac{1}{2 \lambda_{n}} d^{2}\left(y, x_{n}\right)\right] \\
y_{n}=\alpha_{n} w \oplus\left(1-\alpha_{n}\right) z_{n} \\
x_{n+1}=\left(1-\beta_{n}\right) x_{n} \oplus \beta_{n} T^{n} y_{n}
\end{array}\right.
$$

where $\left\{\alpha_{n}\right\}_{n=1}^{\infty} \subset(0,1),\left\{\beta_{n}\right\}_{n=1}^{\infty} \subset[c, d] \subset(0,1)$ satisfying

$$
\lim _{n \rightarrow \infty} \alpha_{n}=0, \quad \sum_{n=1}^{\infty} \alpha_{n}=\infty, \quad \lim _{n \rightarrow \infty} \frac{u_{n}}{\alpha_{n}}=0, \quad \lim _{n \rightarrow \infty} \frac{v_{n}}{\alpha_{n}}=0 .
$$

Assume there exists constant $M>0$, such that $\zeta(r) \leq M r, \forall r \geq 0$. Then $\left\{x_{n}\right\}_{n=1}^{\infty}$ converges strongly to $\Omega$.

P r o o f. Let $p \in \Omega$ and $f(p) \leq f(y), \quad \forall y \in C$. Therefore we obtain

$$
f(p)+\frac{1}{2 \lambda_{n}} d^{2}(p, p) \leq f(y)+\frac{1}{2 \lambda_{n}} d^{2}(y, p), \quad \forall y \in C,
$$


hence $p=J_{\lambda_{n}} p, \forall n \geq 1$. Indeed $z_{n}=J_{\lambda_{n}} x_{n}$ and $J_{\lambda_{n}}$ is nonexpansive [17]. Thus

$$
d\left(z_{n}, p\right)=d\left(J_{\lambda_{n}} x_{n}, J_{\lambda_{n}} p\right) \leq d\left(x_{n}, p\right) .
$$

Let $\delta_{n}:=\alpha_{n} \beta_{n}\left(1+u_{n} M\right)$. Since there exists $N_{0}>0$ such that

$$
\frac{u_{n}}{\alpha_{n}} \leq \frac{\epsilon\left(1+u_{n} M\right)}{M}, \quad \frac{v_{n}}{\alpha_{n}} \leq\left(1+u_{n} M\right)
$$

for all $n \geq N_{0}$ and for some $\epsilon>0$ satisfying $0 \leq(1-\epsilon) \delta_{n} \leq 1$. For any point $p \in \Omega$ and $n \geq N_{0}$, then we have from (3.1) and from Lemma 1 that

$$
\begin{aligned}
d\left(x_{n+1}, p\right)= & d\left(\left(1-\beta_{n}\right) x_{n} \oplus \beta_{n} T^{n} y_{n}, p\right) \\
\leq & \left(1-\beta_{n}\right) d\left(x_{n}, p\right)+\beta_{n} d\left(T^{n} y_{n}, p\right) \\
\leq & \left(1-\beta_{n}\right) d\left(x_{n}, p\right)+\beta_{n}\left(1+M u_{n}\right) d\left(y_{n}, p\right)+\beta_{n} v_{n} \\
= & \left(1-\beta_{n}\right) d\left(x_{n}, p\right)+\beta_{n}\left(1+M u_{n}\right)\left[d\left(\alpha_{n} w \oplus\left(1-\alpha_{n}\right) z_{n}, p\right)\right]+\beta_{n} v_{n} \\
\leq & \left(1-\beta_{n}\right) d\left(x_{n}, p\right)+\alpha_{n} \beta_{n}\left(1+M u_{n}\right) d(w, p)+\beta_{n}\left(1-\alpha_{n}\right)\left(1+M u_{n}\right) d\left(z_{n}, p\right)+\beta_{n} v_{n} \\
\leq & {\left[1-\beta_{n}+\beta_{n}\left(1-\alpha_{n}\right)\left(1+M u_{n}\right)\right] d\left(x_{n}, p\right) } \\
& +\alpha_{n} \beta_{n}\left(1+M u_{n}\right) d(w, p)+\beta_{n} v_{n} \\
\leq & {\left[1-(1-\epsilon) \delta_{n}\right] d\left(x_{n}, p\right)+\delta_{n}(1-\epsilon) \frac{(d(w, p)+1)}{(1-\epsilon)} } \\
\leq & \max \left\{d\left(x_{n}, p\right), \frac{(d(w, p)+1)}{(1-\epsilon)}\right\} .
\end{aligned}
$$

Thus, by induction

$$
d\left(x_{n}, p\right) \leq \max \left\{d\left(x_{N_{0}}, p\right), \frac{(d(w, p)+1)}{(1-\epsilon)}\right\}, \quad \forall n \geq N_{0}
$$

It implies that $\left\{x_{n}\right\}$ is bounded, it follows that $\left\{y_{n}\right\}$ and $\left\{z_{n}\right\}$ are also bounded. Furthermore, from (3.1) and Lemma 2 and letting $\bar{u}_{n}:=2 M u_{n}+u_{n}^{2}$, we obtain

$$
\begin{aligned}
d^{2}\left(x_{n+1}, p\right)= & d^{2}\left(\left(1-\beta_{n}\right) x_{n} \oplus \beta_{n} T^{n} y_{n}, p\right) \\
\leq & \left(1-\beta_{n}\right) d^{2}\left(x_{n}, p\right)+\beta_{n} d^{2}\left(T^{n} y_{n}, p\right)-\beta_{n}\left(1-\beta_{n}\right) d^{2}\left(x_{n}, T^{n} y_{n}\right) \\
\leq & \left(1-\beta_{n}\right) d^{2}\left(x_{n}, p\right)+\beta_{n}\left(\left(1+M u_{n}\right) d\left(y_{n}, p\right)+v_{n}\right)^{2}-\beta_{n}\left(1-\beta_{n}\right) d^{2}\left(x_{n}, T^{n} y_{n}\right) \\
= & \left(1-\beta_{n}\right) d^{2}\left(x_{n}, p\right)+\beta_{n}\left(1+M \bar{u}_{n}\right) d^{2}\left(y_{n}, p\right)+\beta_{n} v_{n}\left[2\left(1+M u_{n}\right) d\left(y_{n}, p\right)+v_{n}\right] \\
& -\beta_{n}\left(1-\beta_{n}\right) d^{2}\left(x_{n}, T^{n} y_{n}\right),
\end{aligned}
$$

also from Lemma 3, we have

$$
\begin{aligned}
d^{2}\left(y_{n}, p\right) & =d^{2}\left(\alpha_{n} w \oplus\left(1-\alpha_{n}\right) z_{n}, p\right) \\
& \leq \alpha_{n}^{2} d^{2}(w, p)+\left(1-\alpha_{n}\right)^{2} d^{2}\left(z_{n}, p\right)+2 \alpha_{n}\left(1-\alpha_{n}\right)\left\langle\overrightarrow{w p}, \overrightarrow{z_{n} p}\right\rangle \\
& \leq \alpha_{n}^{2} d^{2}(w, p)+\left(1-\alpha_{n}\right) d^{2}\left(z_{n}, p\right)+2 \alpha_{n}\left(1-\alpha_{n}\right)\left\langle\overrightarrow{w p}, \overrightarrow{z_{n} p}\right\rangle \\
& \leq \alpha_{n}^{2} d^{2}(w, p)+\left(1-\alpha_{n}\right) d^{2}\left(x_{n}, p\right)+2 \alpha_{n}\left(1-\alpha_{n}\right)\left\langle\overrightarrow{w p}, \overrightarrow{z_{n} p}\right\rangle
\end{aligned}
$$

From (3.2) and (3.4) and the fact that $\left\{y_{n}\right\}$ is bounded, we have that there exists $D>0$ such that 
for any $n \geq N_{0}, d\left(y_{n}, p\right) \leq D$ and letting $\theta_{n}:=\alpha_{n} \beta_{n}$, we obtain

$$
\begin{aligned}
d^{2}\left(x_{n+1}, p\right) \leq & \left(1-\beta_{n}\right) d^{2}\left(x_{n}, p\right)+\beta_{n}\left[\alpha_{n}^{2} d^{2}(w, p)+\left(1-\alpha_{n}\right) d^{2}\left(x_{n}, p\right)+2 \alpha_{n}\left(1-\alpha_{n}\right)\left\langle\overrightarrow{w p}, \overrightarrow{z_{n} \vec{p}}\right\rangle\right] \\
& +\beta_{n} \bar{u}_{n} d^{2}\left(y_{n}, p\right) \beta_{n} v_{n}\left[2\left(1+M u_{n}\right) d\left(y_{n}, p\right)+v_{n}\right]-\beta_{n}\left(1-\beta_{n}\right) d^{2}\left(x_{n}, T^{n} y_{n}\right) \\
\leq & \left(1-\theta_{n}\right) d^{2}\left(x_{n}, p\right)+\theta_{n}\left[\alpha_{n} d^{2}(w, p)+\left(1-\alpha_{n}\right)\left\langle\overrightarrow{w p}, \overrightarrow{z_{n} p}\right\rangle\right] \\
& +\beta_{n}\left[\bar{u}_{n} D^{2}+2 D v_{n}\left(1+M u_{n}\right)+v_{n}^{2}\right]-\beta_{n}\left(1-\beta_{n}\right) d^{2}\left(x_{n}, T^{n} y_{n}\right) \\
\leq & \left(1-\theta_{n}\right) d^{2}\left(x_{n}, p\right)+\theta_{n}\left[\alpha_{n} d^{2}(w, p)+\left(1-\alpha_{n}\right)\left\langle\overrightarrow{w p}, \overrightarrow{z_{n} p}\right\rangle\right] \\
& +\beta_{n}\left[\bar{u}_{n} D^{2}+2 D v_{n}\left(1+M u_{n}\right)+v_{n}^{2}\right] .
\end{aligned}
$$

To complete the proof, we have to consider the following two cases.

Case 1. Suppose $\left\{d\left(x_{n}, p\right)\right\}$ is non-increasing, then $\left\{d\left(x_{n}, p\right)\right\}$ is convergent, from (3.5) and boundedness of $\left\{z_{n}\right\}$, then there exists $D_{1}>0$ such that for any $n \geq N_{0}, d\left(z_{n}, p\right) \leq D_{1}$, thus

$$
\begin{aligned}
\beta_{n}\left(1-\beta_{n}\right) d^{2}\left(x_{n}, T^{n} y_{n}\right) \leq & d^{2}\left(x_{n}, p\right)-d^{2}\left(x_{n+1}, p\right) \\
& +\theta_{n}\left[\alpha_{n} d^{2}(w, p)+2\left(1-\alpha_{n}\right)\left\langle\overrightarrow{w p}, \overrightarrow{z_{n} p}\right\rangle-d^{2}\left(x_{n}, p\right)\right] \\
& +\beta_{n}\left[\bar{u}_{n} D^{2}+2 D v_{n}\left(1+M u_{n}\right)+v_{n}^{2}\right] \\
\leq & d^{2}\left(x_{n}, p\right)-d^{2}\left(x_{n+1}, p\right) \\
& +\theta_{n}\left[\alpha_{n} d^{2}(w, p)+2\left(1-\alpha_{n}\right) D_{1} d(w, p)-d^{2}\left(x_{n}, p\right)\right] \\
& +\beta_{n}\left[\bar{u}_{n} D^{2}+2 D v_{n}\left(1+M u_{n}\right)+v_{n}^{2}\right],
\end{aligned}
$$

which implies that

$$
\beta_{n}\left(1-\beta_{n}\right) d^{2}\left(x_{n}, T^{n} y_{n}\right) \rightarrow 0 \quad \text { as } \quad n \rightarrow \infty,
$$

hence

$$
\lim _{n \rightarrow \infty} d\left(x_{n}, T^{n} y_{n}\right)=0
$$

and from (3.1), we have

$$
d\left(y_{n}, z_{n}\right) \leq \alpha_{n} d\left(w, z_{n}\right)+\left(1-\alpha_{n}\right) d\left(z_{n}, z_{n}\right) \rightarrow 0 \quad \text { as } \quad n \rightarrow \infty,
$$

also from (3.1) and (3.8), we obtain

$$
d\left(x_{n+1}, x_{n}\right) \leq\left(1-\beta_{n}\right) d\left(x_{n}, x_{n}\right)+\beta_{n} d\left(T^{n} y_{n}, x_{n}\right) \rightarrow 0 \quad \text { as } \quad n \rightarrow \infty .
$$

Furthermore from Lemma 6, we see that

$$
\frac{1}{2 \lambda_{n}} d^{2}\left(z_{n}, p\right)-\frac{1}{2 \lambda_{n}} d^{2}\left(x_{n}, p\right)+\frac{1}{2 \lambda_{n}} d^{2}\left(x_{n}, z_{n}\right) \leq f(p)-f\left(z_{n}\right),
$$

since $f(p) \leq f\left(z_{n}\right)$ for all $n \geq 1$, it follows that

$$
d^{2}\left(x_{n}, z_{n}\right) \leq d^{2}\left(x_{n}, p\right)-d^{2}\left(z_{n}, p\right) .
$$

But from (3.2) and (3.3), we obtain

$$
\begin{gathered}
d^{2}\left(x_{n+1}, p\right) \leq\left(1-\beta_{n}\right) d^{2}\left(x_{n}, p\right)+\beta_{n}\left[\alpha_{n}^{2} d^{2}(w, p)+\left(1-\alpha_{n}\right) d^{2}\left(z_{n}, p\right)+2 \alpha_{n}\left(1-\alpha_{n}\right)\left\langle\overrightarrow{w p}, \overrightarrow{z_{n} p}\right\rangle\right] \\
+\beta_{n}\left[2 v_{n}\left(1+M u_{n}\right) d\left(y_{n}, p\right)+v_{n}^{2}+\bar{u}_{n} d^{2}\left(y_{n}, p\right)\right]
\end{gathered}
$$


therefore, from (2.1) and boundedness of $\left\{z_{n}\right\}$, we obtain

$$
\begin{aligned}
d^{2}\left(x_{n}, p\right) \leq & \frac{1}{\beta_{n}}\left(d^{2}\left(x_{n}, p\right)-d^{2}\left(x_{n+1}, p\right)\right)+\alpha_{n}^{2} d^{2}(w, p)+\left(1-\alpha_{n}\right) d^{2}\left(z_{n}, p\right) \\
& +2 \alpha_{n}\left(1-\alpha_{n}\right) D_{1} d(w, p)+2 D v_{n}\left(1+M u_{m}\right)+\bar{u}_{n} D^{2}
\end{aligned}
$$

from (3.11) and (3.12), we obtain

$$
\begin{gathered}
d^{2}\left(x_{n}, z_{n}\right) \leq \frac{1}{\beta_{n}}\left(d^{2}\left(x_{n}, p\right)-d^{2}\left(x_{n+1}, p\right)\right)+\alpha_{n}\left(\alpha_{n} d^{2}(w, p)-d^{2}\left(z_{n}, p\right)\right) \\
+2 \alpha_{n}\left(1-\alpha_{n}\right) D_{1} d(w, p)+2 D v_{n}\left(1+M u_{m}\right)+\bar{u}_{n} D^{2}
\end{gathered}
$$

since $\left\{x_{n}\right\}$ and $\left\{z_{n}\right\}$ are bounded and $\left\{d\left(x_{n}, p\right)\right\}$ is non-increasing sequence, it follows from that

$$
\lim _{n \rightarrow \infty} d\left(x_{n}, z_{n}\right)=0
$$

from (3.9) and (3.13), we obtain

$$
\lim _{n \rightarrow \infty} d\left(x_{n}, y_{n}\right)=0
$$

and

$$
d\left(y_{n}, T^{n} y_{n}\right) \leq d\left(y_{n}, x_{n}\right)+d\left(x_{n}, T^{n} y_{n}\right) \rightarrow 0 \quad \text { as } \quad n \rightarrow \infty,
$$

also from (3.14) and (3.15), we obtain

$$
\begin{aligned}
d\left(x_{n}, T^{n} x_{n}\right) & \leq d\left(x_{n}, y_{n}\right)+d\left(y_{n}, T^{n} y_{n}\right)+d\left(T^{n} y_{n}, T^{n} x_{n}\right) \\
& \leq\left(2+M u_{n}\right) d\left(x_{n}, y_{n}\right)+d\left(y_{n}, T^{n} y_{n}\right)+v_{n} \rightarrow 0
\end{aligned}
$$

as $n \rightarrow \infty$. Observe also that since $T$ is uniformly $L$-Lipschitzian, we have

$$
\begin{aligned}
d\left(x_{n}, T x_{n}\right) & \leq d\left(x_{n}, T^{n} x_{n}\right)+d\left(T^{n} x_{n}, T x_{n}\right) \\
& \leq d\left(x_{n}, T^{n} x_{n}\right)+L d\left(T^{n-1} x_{n}, x_{n}\right) \\
& \leq d\left(x_{n}, T^{n} x_{n}\right)+L\left[d\left(T^{n-1} x_{n}, T^{n-1} x_{n-1}\right)+d\left(T^{n-1} x_{n-1}, x_{n-1}\right)+d\left(x_{n-1}, x_{n}\right)\right] \\
& \leq d\left(x_{n}, T^{n} x_{n}\right)+L d\left(T^{n-1} x_{n-1}, x_{n-1}\right)+L(1+L) d\left(x_{n}, x_{n-1}\right),
\end{aligned}
$$

it follows from (3.10) and (3.16) that

$$
\lim _{n \rightarrow \infty} d\left(x_{n}, T x_{n}\right)=0,
$$

from (3.14) and (3.17), we obtain

$$
\begin{aligned}
d\left(y_{n}, T y_{n}\right) & \leq d\left(y_{n}, x_{n}\right)+d\left(x_{n}, T x_{n}\right)+d\left(T x_{n}, T y_{n}\right) \\
& \leq(1+L) d\left(y_{n}, x_{n}\right)+d\left(x_{n}, T x_{n}\right) \rightarrow 0
\end{aligned}
$$

as $n \rightarrow \infty$. Also since $\lambda_{n} \geq \lambda>0$, from Lemma 7 , we obtain

$$
\begin{aligned}
d\left(J_{\lambda} x_{n}, J_{\lambda_{n}} x_{n}\right) & =d\left(J_{\lambda} x_{n}, J_{\lambda}\left(\frac{\lambda_{n}-\lambda}{\lambda_{n}} J_{\lambda_{n}} x_{n} \oplus \frac{\lambda}{\lambda_{n}} x_{n}\right)\right) \\
& \leq d\left(x_{n},\left(1-\frac{\lambda}{\lambda_{n}}\right) J_{\lambda_{n}} x_{n} \oplus \frac{\lambda}{\lambda_{n}} x_{n}\right) \\
& \leq\left(1-\frac{\lambda}{\lambda_{n}}\right) d\left(x_{n}, z_{n}\right) \rightarrow 0
\end{aligned}
$$


as $n \rightarrow \infty$, hence

$$
d\left(x_{n}, J_{\lambda} x_{n}\right) \leq d\left(x_{n}, z_{n}\right)+d\left(z_{n}, J_{\lambda} x_{n}\right) \rightarrow 0 \quad \text { as } \quad n \rightarrow \infty .
$$

Moreover, since $\left\{x_{n}\right\}$ is bounded and $X$ is a complete CAT(0) space, by Lemma 4 we choose a subsequence $\left\{x_{n_{i}}\right\}$ of $\left\{x_{n}\right\}$ such that $\triangle-\lim x_{n_{i}}=v$, where $v:=P_{\Omega}(w)$. Then, from (3.15), (3.18), Lemma 8 and the fact that $J_{\lambda}$ is nonexpansive [17], we have $v \in F(T)$, also from Lemma 5 , we have

$$
\lim \sup \left\langle\overrightarrow{w v}, \overrightarrow{x_{n} v}\right\rangle \leq 0
$$

Furthermore, since

$$
\begin{aligned}
\left\langle\overrightarrow{w v}, \overrightarrow{z_{n}} \vec{v}\right\rangle & =\left\langle\overrightarrow{w v}, \overrightarrow{z_{n} x_{n}}\right\rangle+\left\langle\overrightarrow{w v}, \overrightarrow{x_{n} v}\right\rangle \\
& \leq d(w, v) d\left(z_{n}, x_{n}\right)+\left\langle\overrightarrow{w v}, \overrightarrow{x_{n} v}\right\rangle
\end{aligned}
$$

it follows from (3.13) and (3.19) that

$$
\lim \sup \left\langle\overrightarrow{w v}, \overrightarrow{z_{n} v}\right\rangle \leq 0
$$

Thus, now putting $v:=p$ in inequality (3.6), we get that, for $n \geq N_{0}$

$$
\begin{gathered}
d^{2}\left(x_{n+1}, v\right) \leq\left(1-\theta_{n}\right) d^{2}\left(x_{n}, v\right)+\theta_{n}\left[\alpha_{n} d^{2}(w, v)+\left(1-\alpha_{n}\right)\left\langle\overrightarrow{w v}, \overrightarrow{z_{n}} \vec{v}\right\rangle\right] \\
+\beta_{n}\left[\bar{u}_{n} D^{2}+2 D v_{n}\left(1+M u_{n}\right)+v_{n}^{2}\right] .
\end{gathered}
$$

Hence

$$
d^{2}\left(x_{n+1}, v\right) \leq\left(1-\theta_{n}\right) d^{2}\left(x_{n}, v\right)+\theta_{n} \sigma_{n}+\gamma_{n}
$$

where

$$
\sigma_{n}:=\alpha_{n} d^{2}(w, v)+\left(1-\alpha_{n}\right)\left\langle\overrightarrow{w v}, \overrightarrow{z_{n}} \vec{v}\right\rangle, \quad \gamma_{n}:=\beta_{n}\left[\bar{u}_{n} D^{2}+2 D v_{n}\left(1+M u_{n}\right)+v_{n}^{2}\right],
$$

it follows from Lemma 10 that $d\left(x_{n}, v\right) \rightarrow 0$ as $n \rightarrow \infty$. Consequently, $x_{n} \rightarrow v$.

Case 2. Suppose that $\left\{d\left(x_{n}, p\right)\right\}_{n \geq 1}$ is non-decreasing sequence. Then, there exists a subsequence $\left\{n_{i}\right\}$ of $\{n\}$ such that

$$
d\left(x_{n_{i}}, p\right)<d\left(x_{n_{i}+1}, p\right)
$$

for all $i \in \mathbb{N}$. Then, by Lemma 9 , there exists an increasing sequence $\left\{m_{j}\right\}_{j \geq 1}$ such that $m_{j} \rightarrow \infty$, $d\left(x_{m_{j}}, p\right) \leq d\left(x_{m_{j}+1}, p\right)$ and $d\left(x_{j}, p\right) \leq d\left(x_{m_{j}+1}, p\right)$ for all $j \geq 1$. Then from (3.7), we obtain

$$
\begin{aligned}
\beta_{m_{j}}\left(1-\beta_{m_{j}}\right) d^{2}\left(x_{m_{j}}, T^{m_{j}} y_{m_{j}}\right) \leq & d^{2}\left(x_{m_{j}}, p\right)-d^{2}\left(x_{m_{j}+1}, p\right) \\
& +\theta_{m_{j}}\left[\alpha_{m_{j}} d^{2}(w, p)+2\left(1-\alpha_{m_{j}}\right)\left\langle\overrightarrow{w p}, \overrightarrow{z_{m_{j}}} p\right\rangle-d^{2}\left(x_{m_{j}}, p\right)\right] \\
& +\beta_{m_{j}}\left[\bar{u}_{m_{j}} D^{2}+2 D v_{m_{j}}\left(1+M u_{m_{j}}\right)+v_{m_{j}}^{2}\right] \\
\leq & d^{2}\left(x_{m_{j}}, p\right)-d^{2}\left(x_{m_{j}+1}, p\right) \\
& +\theta_{m_{j}}\left[\alpha_{m_{j}} d^{2}(w, p)+2\left(1-\alpha_{m_{j}}\right) D_{1} d(w, p)-d^{2}\left(x_{m_{j}}, p\right)\right] \\
& +\beta_{m_{j}}\left[\bar{u}_{m_{j}} D^{2}+2 D v_{m_{j}}\left(1+M u_{m_{j}}\right)+v_{m_{j}}^{2}\right] .
\end{aligned}
$$

This implies $d\left(x_{m_{j}}, T^{m_{j}} y_{m_{j}}\right) \rightarrow 0$ as $j \rightarrow \infty$. Thus, as in Case 1, we obtain that $d\left(x_{m_{j}}, T x_{m_{j}}\right) \rightarrow 0$ and $d\left(x_{m_{j}}, J_{\lambda} x_{m_{j}}\right) \rightarrow 0$ as $j \rightarrow \infty$ and also following the same argument in Case 1, we get $\lim \sup \left\langle\overrightarrow{w v}, \overrightarrow{z_{m_{j}}} \vec{v}\right\rangle \leq 0$, where $v:=P_{\Omega}(w)$. Also from (3.20), we obtain that,

$$
\begin{gathered}
d^{2}\left(x_{m_{j}+1}, v\right) \leq\left(1-\theta_{n}\right) d^{2}\left(x_{m_{j}}, v\right)+\theta_{m_{j}}\left[\alpha_{m_{j}} d^{2}(w, v)+\left(1-\alpha_{m_{j}}\right)\left\langle\overrightarrow{w v}, \overrightarrow{z_{m_{j}}} \vec{v}\right\rangle\right] \\
+\beta_{m_{j}}\left[\bar{u}_{m_{j}} D^{2}+2 D v_{m_{j}}\left(1+M u_{m_{j}}\right)+v_{m_{j}}^{2}\right] .
\end{gathered}
$$


Since $d^{2}\left(x_{m_{j}}, v\right) \leq d^{2}\left(x_{m_{j}+1}, v\right)$, it follows that

$$
\begin{aligned}
& \theta_{m_{j}} d^{2}\left(x_{m_{j}}, v\right) \leq d^{2}\left(x_{m_{j}}, v\right)-d^{2}\left(x_{m_{j}+1}, v\right)+\theta_{m_{j}}\left[\alpha_{m_{j}} d^{2}(w, v)+\left(1-\alpha_{m_{j}}\right)\left\langle\overrightarrow{w v}, \overrightarrow{z_{m_{j}}} \overrightarrow{ }\right\rangle\right] \\
& +\beta_{m_{j}}\left[\bar{u}_{m_{j}} D^{2}+2 D v_{m_{j}}\left(1+M u_{m_{j}}\right)+v_{m_{j}}^{2}\right] \\
& \leq \theta_{m_{j}}\left[\alpha_{m_{j}} d^{2}(w, v)+\left(1-\alpha_{m_{j}}\right)\left\langle\overrightarrow{w v}, \overrightarrow{z_{m_{j}}}\right\rangle\right] \\
& +\beta_{m_{j}}\left[\bar{u}_{m_{j}} D^{2}+2 D v_{m_{j}}\left(1+M u_{m_{j}}\right)+v_{m_{j}}^{2}\right] \text {. }
\end{aligned}
$$

In particular, since $\theta_{m_{j}}>0$, we get

$$
d^{2}\left(x_{m_{j}}, v\right) \leq\left[\alpha_{m_{j}} d^{2}(w, v)+\left(1-\alpha_{m_{j}}\right)\left\langle\overrightarrow{w v}, \overrightarrow{z_{m_{j}}}\right\rangle\right]+\left[\frac{\bar{u}_{m_{j}}}{\alpha_{m_{j}}} D^{2}+2 D \frac{v_{m_{j}}}{\alpha_{m_{j}}}\left(1+M u_{m_{j}}\right)+v_{m_{j}} \frac{v_{m_{j}}}{\alpha_{m_{j}}}\right] .
$$

Then, since $\lim \sup \left\langle\overrightarrow{w v}, \overrightarrow{x_{m_{j}}} \vec{v}\right\rangle \leq 0$ and the fact that $\alpha_{m_{j}} \rightarrow 0$ as $j \rightarrow \infty$ and

$$
\lim _{j \rightarrow \infty} \frac{u_{m_{j}}}{\alpha_{m_{j}}}=0, \quad \lim _{j \rightarrow \infty} \frac{v_{m_{j}}}{\alpha_{m_{j}}}=0
$$

we obtain that $d\left(x_{m_{j}}, v\right) \rightarrow 0$ as $j \rightarrow \infty$. This together with (3.21) give $d\left(x_{m_{j}+1}, v\right) \rightarrow 0$ as $j \rightarrow \infty$. But $d\left(x_{j}, v\right) \leq d\left(x_{m_{j}+1}, v\right)$, for all $j \geq 1$, thus we obtain that $x_{j} \rightarrow v$. Therefore, from the above two cases, we can conclude that $\left\{x_{n}\right\}_{n=1}^{\infty}$ converges strongly to an element of $\Omega$ and the proof is complete.

\section{Conclusion}

In this work, we study a modified Halpern-type proximal point algorithm for finding the minimizer of a convex lower semi-continuous function which is also a fixed point of total asymptotically nonexpansive mapping. Under some appropriate assumption, we have obtained a strong convergence theorem for the proposed algorithm in the framework of a complete CAT $(0)$ space.

\section{REFERENCES}

1. Alber Ya. I., Chidume C. E., Zegeye H. Approximating fixed points of total asymptotically nonexpansive mappings. Fixed Point Theory Appl., 2006. Art. no. 10673. P. 1-20. DOI: 10.1155/FPTA/2006/10673

2. Ahmad I., Ahmad M. An implicit viscosity technique of nonexpansive mapping in CAT(0) spaces. Open J. Math. Anal., 2017. Vol. 1. P. 1-12. DOI: 10.30538/psrp-oma2017.0001

3. Agarwal R.P., O'Regan D., Sahu D. R. Iterative construction of fixed points of nearly asymptotically nonexpansive mappings. J. Nonlinear Convex Anal., 2007. Vol. 8, No. 1. P. 61-79.

4. Ambrosio L., Gigli N., Savare G. Gradient Flows in Metric Spaces and in the Space of Probability Measures, 2nd ed. Lectures in Mathematics ETH Zürich. Basel: Birkhäuser, 2008. 334 p. DOI: $10.1007 / 978-3-7643-8722-8$

5. Ariza-Ruiz D., Leustean L., López-Acedo G. Firmly nonexpansive mappings in classes of geodesic spaces. Trans. Amer. Math. Soc., 2014. Vol. 366. No. 8. P. 4299-4322. DOI: 10.1090/S0002-9947-2014-05968-0

6. Bačák M. The proximal point algorithm in metric spaces. Israel J. Math., 2013. Vol. 194. P. 689-701. DOI: $10.1007 / \mathrm{s} 11856-012-0091-3$

7. Berg I. D., Nikolaev I. G. Quasilinearization and curvature of Aleksandrov spaces. Geom. Dedicata, 2008. Vol. 133. P. 195-218. DOI: 10.1007/s10711-008-9243-3

8. Bonyah E., Ahmad M., Ahmad I. On the viscosity rule for common fixed points of two nonexpansive mappings in CAT(0) spaces. Open J. Math. Sci., 2018. Vol. 2. No. 1. P. 39-55. DOI: 10.30538/oms2018.0016

9. Bridson M. R., Häfliger A. Metric Spaces of Nonpositive Curvature. Grundlehren Math. Wiss., vol. 319. Berlin, Heidelberg: Springer-Verlag, 1999. 643 p. DOI: 10.1007/978-3-662-12494-9

10. Burago D., Burago Yu., Ivanov S. A Course in Metric Geometry. Grad. Stud. Math., vol. 33. Providence, RI: A.M.S., 2001. 415 p. 
11. Chang S.-S., Wang L., Joseph Lee H. W., Chan C.K., Yang L. Demiclosed principle and $\triangle$-convergence theorems for total asymptotically nonexpansive mappings in CAT(0) spaces. Appl. Math. Comput., 2012. Vol. 219, No. 5. P. 2611-2617. DOI: 10.1016/j.amc.2012.08.095

12. Chang S.-S., Yao J.-C., Wang L., Qin L. J. Some convergence theorems involving proximal point and common fixed points for asymptotically nonexpansive mappings in CAT(0) spaces. Fixed Point Theory Appl., 2016. Art. no. 68. P. 1-11. DOI: 10.1186/s13663-016-0559-7

13. Cholamjiak P., Abdou A.A., Cho Y.J. Proximal point algorithms involving fixed points of nonexpansive mappings in CAT(0) spaces. Fixed Point Theory Appl., 2015. Art. no. 227. P. 1-13. DOI: $10.1186 / \mathrm{s} 13663-015-0465-4$

14. Dehghan H., Rooin J. A Characterization of Metric Projection in CAT(0) spaces. 2012.3 p. arXiv: 1311.4174 [math.FA]

15. Dhompongsa S., Kirk W. A., Sims B. Fixed points of uniformly lipschitzian mappings. Nonlinear Anal., 2006. Vol. 65, No. 4. P. 762-772. DOI: 10.1016/j.na.2005.09.044

16. Dhompongsa S., Panyanak B. On $\triangle$-convergence theorems in CAT(0) spaces. Comput. Math. Appl., 2008. Vol. 56, No. 10. P. 2572-2579. DOI: 10.1016/j.camwa.2008.05.036

17. Jost J. Convex functionals and generalized harmonic maps into spaces of non positive curvature. Comment. Math. Helv., 1995. Vol. 70. P. 659-673. DOI: 10.1007/BF02566027

18. Güler O. On the convergence of the proximal point algorithm for convex minimization. SIAM J. Control Optim., 1991. Vol. 29, No. 2. P. 403-419. DOI: 10.1137/0329022

19. Kakavandi B. A. Weak topologies in complete CAT(0) metric spaces. Proc. Amer. Math. Soc., 2012. Vol. 141, No. 3. P. 1029-1039. URL: https://www.jstor.org/stable/23558440

20. Kamimura S., Takahashi W. Approximating solutions of maximal monotone operators in Hilbert spaces. J. Approx. Theory, 2000. Vol. 106, No. 2. P. 226-240. DOI: 10.1006/jath.2000.3493

21. Kang S. M., Haq A. U., Nazeer W., Ahmad I., Ahmad M. Explicit viscosity rule of nonexpansive mappings in CAT(0) spaces. J. Comput. Anal. Appl., 2019. Vol. 27, No. 6. P. 1034-1043.

22. Kirk W.A. Geodesic geometry and fixed point theory. In: Seminar of Mathematical Analysis, Malaga/Seville, 2002/2003, Álvares D.G., Acelo G.L., Caro R.V. (eds.), vol. 64. P. 195-225.

23. Kirk W. A.Geodesic geometry and fixed point theory, II. In: Int. Conf. on Fixed Point Theory and Applications, Yokohama Publ., Yokohama, Japan, 2004. P. 113-142.

24. Kirk W. A., Panyanak B. A concept of convergence in geodesic spaces. Nonlinear Anal., 2008. Vol. 68, No. 12. P. 3689-3696. DOI: 10.1016/j.na.2007.04.011

25. Maingé P. E. Strong convergence of projected subgradient methods for nonsmooth and nonstrictly convex minimization. Set-Valued Anal., 2008. Vol. 16, No. 7-8. P. 899-912. DOI: 10.1007/s11228-008-0102-z

26. Martinet B. Régularisation d'inéquations variationnelles par approximations successives. Rev. Fr. Inform. Rech. Opér., 1970. Vol. 4, No. R3. P. 154-158. (in France)

27. Mayer U. F. Gradient flows on nonpositively curved metric spaces and harmonic maps. Commun. Anal. Geom. 1998. Vol. 6, No. 2. P. 199-253.

28. Rockafellar R. T. Monotone operators and the proximal point algorithm. SIAM J. Control Optim., 1976. Vol. 14, No. 5. P. 877-898. DOI: /10.1137/0314056

29. Suparatulatorn R., Cholamjiak P., Suantai S. On solving the minimization problem and the fixed-point problem for nonexpansive mappings in CAT(0) spaces. Optim. Methods Softw., 2017. Vol. 32, No. 1. P. 182-192. DOI: 10.1080/10556788.2016.1219908

30. Xu H. K. Iterative algorithms for nonlinear operators. J. Lond. Math. Soc. (2), 2002. Vol. 66, No. 1. P. 240-256. DOI: 10.1112/S0024610702003332 